\title{
January updates: people and places
}

\section{Global 3Rs awardees}

AAALAC International and the IQ

Consortrium have announced the winners of the 2018 Global 3Rs Awards, an annual program that recognizes contributions to the advancement of the 3 Rs by a researcher or researchers in Europe, North America, and the Pacific Rim. The awards were presented at the $69^{\text {th }}$ National Meeting of American Association of Laboratory Animal Science in Baltimore, Maryland. Each awardee received $\$ 5000$.

The European winner was Esther Rodriquez-Villegas from Imperial College London. She was recognized for her 2017 paper, "TaiNi: Maximizing research output whilst improving animals' welfare in neurophysiology experiments," published in Scientific Reports about the TaiNi system, a wireless neural monitoring device that is light weight (just $1.5 \mathrm{~g}$ ) and can continuously record 16 channels of electrophysiological activity for over three days. The system is an improvement over previous devices that were often heavier, tethered, and did not last as long, thus requiring more animal handling.

For North America, Kelly Coleman from Medtronic Physiological Research Laboratories in Minneapolis, Minnesota was recognized for his 2018 paper, "Round robin study to evaluate the reconstructed human epidermis (RhE) model as an in vitro skin irritation test for detection of irritant activity in medical device extracts," published in Toxicology In Vitro that evaluated a human epidermis model as an alternative to rabbits for testing the biocompatibility of medical devices.

And from the Pacific Rim, a team of eight researchers from Singapore-Gopu Sriram, Massimo Alberti, Yuri Dancik, Bo Wu, Ruige Wu, Srinivas Ramasamy, Mei Bigliardi-Qi and Zhiping Wang-was recognized for their 2018 paper, "Fullthickness human skin-on-chip with enhanced epidermal morphogenesis and barrier function," published in Materials Today that presented a microfluidic skinon-chip device that can be used for testing drugs and skin care products.

\section{GEMM call}

The Medical Research Council (MRC) in the UK has announced GEMM call 5. GEMMGenome Editing Mice for Medicine-is a service provided by the Mary Lyon Centre. UK researchers can apply for the creation a novel genetically altered mouse line with a point mutation, indel, or deletion mutation through the call. Applications are due by January $18^{\text {th }} 2019$. The winning mouse lines will be publically available to other researchers through the European Mouse Mutant Archive. For more information, visit: https://www.har.mrc.ac.uk/internationalprogrammes/gemms

The MRC has committed $£ 4.5 \mathrm{~m}$ over five years to the GEMM service.

\section{Database 2.0 released at CrownBio} Crown Bioscience has announced the release of MuBase 2.0, an update to the company's online database of murine immuno-oncology models. The company has released additional data to users and also re-designed the user interface of the platform. "This ongoing investment in improving our open access databases demonstrates CrownBio's commitment to providing the most relevant data for the most appropriate in vivo model for each drug discovery program," said Jean Pierre Wery, CEO of Crown Bioscience.

\section{NIAID contract to Charles River}

Charles River Laboratories has been awarded a $\$ 95.7$ million contract from the National Institutes of Health National Institute of Allergy and Infectious Disease (NIH NIAID). The company will manage and staff NIAID's on-site vivarium and provide support for related animal model operations. Charles River staff duties will include providing veterinary care, research animal husbandry, and other technical and logistical support.

James C. Foster, Chairman, President and Chief Executive Officer of Charles River, commented in a press release, "We are very pleased to be awarded this significant contract by NIAID and look forward to establishing a collaborative relationship to support NIAID's critical biomedical research programs across a range of scientific disciplines."
The contract began on September $14^{\text {th }}$ and can be renewed after September 13, 2019 for an additional four years.

\section{Taconic joins GSA contract schedule}

Taconic Biosciences has announced a new partnership with Government Scientific Source (GSS), a Reston, Virginia-based company contracted by the US General Services Administration to provide laboratory equipment to federal, state, and local governments. Taconic animal models will be available to government agencies through the partnership.

"GSS is excited to be partnering with Taconic. We are particularly impressed with vast array of transgenic and germ-free models. The Taconic products are a great fit for our GSS effort to support translational research solutions within the U.S. Federal Government," commented Tony Nardei, director of life science at Government Scientific Source.

\section{Catapulting technology}

The National Centre for the Replacement, Refinement \& Reduction of Animals in Research (NC3Rs) in the United Kingdom has announced a new collaboration with the Medicines Discovery Catapult to help translate 3 Rs research for industry. A new funding scheme, "Technologies to Tools," has been launched by the NC3Rs in support of the collaboration.

Anthony Holmes, NC3Rs Director of Science and Technology, commented: "NC3Rs-funded research represents a very broad, diverse portfolio of technologically advanced, human-relevant approaches. I believe we're only just scratching the surface of the potential for these technologies to drive a paradigm shift in the way the pharmaceutical industry develops and tests new drugs; one where decisions on whether to progress a potential therapeutic are based on data generated in human-relevant systems and not animal models. The collaboration with MDC presents a timely opportunity in maintaining the position of NC3Rs-funded research at the forefront of these endeavours."

Published online: 12 December 2018 https://doi.org/10.1038/s41684-018-0198-1 